\title{
An Association Between Blastocystis Subtypes and Colorectal Cancer Patients: A Significant Different Profile from Non-cancer Individuals
}

\author{
Shaimaa H. Ali ${ }^{1}$ (D) Mousa A. M. Ismail ${ }^{2}$ (1) - Ayman A. El-Badry ${ }^{3}$ () - Enas Y. Abu-Sarea ${ }^{1}$ (1) $\cdot$ Ahmad M. Dewidar $^{4}$. \\ Doaa A. Hamdy ${ }^{1}$ (D)
}

Received: 4 October 2021 / Accepted: 9 December 2021 / Published online: 24 January 2022

(c) The Author(s) under exclusive licence to Witold Stefański Institute of Parasitology, Polish Academy of Sciences 2021

\begin{abstract}
Purpose Blastocystis is a common enteric human parasite of non-conclusive pathogenicity which may be determined by subtype (ST) variation. Colorectal cancer (CRC) is considered one of the primary causes of cancer mortality. Blastocystis ST7 has been shown to reduce beneficial intestinal microbiota and may exacerbate CRC. This study assessed the possible association between Blastocystis STs and CRC in comparison to non-cancer patients.

Material and Methods A total of 200 fecal samples were obtained from CRC (100) and non-CRC (100) individuals attending Beni-Suef University Hospital, Egypt. Blastocystis was searched for in all samples using microscopy and culturing. Positive subculture samples were genetically sequenced and subtyped using conventional polymerase chain reaction (PCR). Blastocystis STs were determined by sequencing and a phylogenetic tree was created. Related patient characteristics and tumor stages were analyzed for association with presence of Blastocystis.

Results Blastocystis was identified in 52\% and $42 \%$ of CRC and non-cancer individuals, respectively. ST1, 2, and 3 were isolated from both cancer and non-cancer individuals; however, for the first time, ST7 was only isolated from CRC stool samples with significant association. Associated patient characteristics were evaluated as predictors.

Conclusion Blastocystosis is highly prevalent in CRC patients, predominantly in the latest CRC grades and stages. To the best of our knowledge, this is the first study to report the identification of Blastocystis ST7 in CRC patients. To determine whether certain STs of Blastocystis are associated with CRC would require further research, including the role played by gut microbiota.
\end{abstract}

Keywords Blastocystis $\cdot$ Subtypes $\cdot$ Colorectal cancer $\cdot$ Egypt

Doaa A. Hamdy

doaahamdypara@gmail.com

Shaimaa H. Ali

shaimaahussien88@yahoo.com

Mousa A. M. Ismail

dr.mousa.abdelgawad@gmail.com

Ayman A. El-Badry

aelbadry@kasralainy.edu.eg; aaelbadry@iau.edu.sa

Enas Y. Abu-Sarea

enasyahia71@yahoo.com

Ahmad M. Dewidar

ahmaddewidar@yahoo.com
1 Department of Medical Parasitology, College of Medicine, Beni-Suef University, Beni Suef, Egypt

2 Department of Medical Parasitology, College of Medicine, Cairo University, Giza, Egypt

3 Department of Microbiology-Medical Parasitology Section, College of Medicine, Imam Abdulrahman Bin Faisal University, Dammam, Saudi Arabia

4 Department of Oncology, College of Medicine, Beni-Suef University, Beni Suef, Egypt 


\section{Introduction}

Blastocystis species (sp.) is one of the most common parasites inhabiting the human colon and encountered in human feaces which has aroused scientific attention due to great controversy about its biology. It is currently considered the most commonly $[1,2]$.

Blastocystis sp. has zoonotic potential with pandemic prevalence representing wide variations between different countries and even between multiple areas in the same country [3]. Generally, the prevalence in developing countries is higher (22.1-100\%) than developed ones $(0.5-23.1 \%)$ [4]. This difference might be explained by differences in level of hygiene standards, exposure to animals, consumption of contaminated food or drink, and unsanitary waste disposal [4].

The non-specific symptoms caused by blastocystosis include nausea, anorexia, vomiting, diarrhea, abdominal pain, flatulence, and weight loss. Blastocystis sp. was even reported to have an important role in enhancing carcinogenesis in Blastocystis sp. infected mice [5].

Many factors may affect Blastocystis sp. infection such as the immune status and age of hosts [6]. Among immunosuppressed individuals, blastocystosis was identified as a clinically relevant enteric infection that led to severe chronic diarrhea [3, 7].

CRC is the fourth most commonly and third most deadly diagnosed cancer in the world, and three-to-four times more prevalent in developed countries than in developing countries [8, 9]. Infectious agents might be responsible for $20 \%$ of CRC [7] and at least one sixth of all cancer cases worldwide [10]. Recent studies have highlighted the substantial role of Blastocystis in CRC, and routine screening for the parasite is recommended [7, 8].

Initiation and progression of CRC were evidenced by involvement of the intestinal microbiota. The role of intestinal microbiota outside the gut, as well as in gut physiology, is well documented. Intestinal microbiota has an essential impact on immune system maturation, most metabolic pathways, and different gastrointestinal diseases, such as inflammatory bowel diseases and CRC [11].

Worldwide distribution of Blastocystis sp. and its genetic diversity had been approved. Genotyping of Blastocystis isolates has received great attention nowadays in an attempt to correlate variance pathogenic behaviors of the parasite to its different STs [1]. Up to date, 17 Blastocystis STs, mostly with low host specificity, have been identified and ten of them were reported in humans, with variable prevalence [12-14].

The current study aimed to investigate and assess the frequency and potential role of Blastocystis infection among CRC patients in comparison to non-cancer individuals in Beni Suef Governorate, Egypt. As well as, to determine the STs of encountered Blastocystis and to evaluate their association potential with CRC.

\section{Material and Methods}

\section{Study Design and Population}

A cross-sectional, hospital-based study was performed on two hundred (200) patients, 100 patients diagnosed with CRC (cases) (cancer group), and 100 apparently healthy (sex- and age-matched) subjects (control) attending the out-patient clinics of Beni-Suef University Hospital for regular checkup or healthy individuals accompanying cancer patients assigned as control (non-cancer) group. Control group was free from any tumors or chronic diseases $(n=100)$. The study was conducted between February 2019 and February 2021. Cases (cancer group) included patients of both sexes, of all ages having CRC $(n=100)$. This group was subdivided into two groups; Group A: $(n=50)$ diagnosed as CRC patients that had not started any anticancer treatment regime and had not received any anti-parasitic medication for the past 2 weeks. Group B: $(n=50)$ patients with CRC receiving radio or chemotherapy. Inclusion criteria included cooperative individuals of all ages and both sexes who agreed to be engaged in the study and were able to provide adequate samples. Exclusion criteria included patients having any type of tumors other than CRC, and patients taking anti-parasitic medications 2 weeks before sample collection. A data collection sheet containing relevant clinical and demographic data was obtained from each participant. Colonoscopy grading of CRC was done according to the American Society of Clinical Oncology [15]. Colorectal Cancer, grading describes how the cancer cells look compared to normal, healthy cells.

\section{Stool Sampling and Processing}

\section{Direct Microscopic Examination and Staining}

Fresh fecal specimens (about 2 gm each) were collected in clean, dry, wide-mouth plastic containers with tight lids. Date of collection, patient's name, and serial number were labeled on each container, and the sample was subdivided into three parts. The first part of the specimen $(50 \mathrm{mg})$ was microscopically examined using saline and iodine wet mounts using $40 \times$ and $100 \times$ objectives. The second part about $(50 \mathrm{mg})$ of each specimen was preserved in $5 \%$ buffered formalin solution followed by staining with modified trichrome stain. Procedure of staining was performed according to Garcia [16]. Microscopic examination of 
stained smears was done using oil immersion and highpower objectives.

\section{Culture and Subculture of Blastocystis sp.}

The third part of the specimen was in vitro cultured on modified Jones' medium for Blastocystis sp. identification [17]. Fifty milligrams from each stool specimen were inoculated into sterile $7 \mathrm{ml} \mathrm{screw}$ caped tubes containing $5 \mathrm{ml}$ Jones' medium and were incubated at $37{ }^{\circ} \mathrm{C}$ for $2-3$ days [18]. Examination of the culture was done after $24,48,72$, and $96 \mathrm{~h}$ by taking one drop from the tube under sterile conditions, with a sterile Pasteur pipette, and examined with low-power $(\times 100)$, then with high-power $(\times 400)$ magnification. The culture was considered negative and discarded if Blastocystis sp. was not detected after $96 \mathrm{~h}$. Meanwhile, $1 \mathrm{ml}$ of the positive culture samples were transferred to fresh medium using sterile Pasteur pipette under complete sterile conditions (under the UVR-Laminar flow hood) for 48-72 h. [19]. Suspensions of Blastocystis were centrifuged at $500 \times \mathrm{g}$ for $5 \mathrm{~min}$. The pellet was resuspended in phosphate buffer saline solution, and the process was repeated five times. The final pellet was stored at $-20^{\circ} \mathrm{C}$ for DNA extraction and further molecular assays [20].

\section{Molecular Identification of Blastocystis sp.}

Blastocystis positive subculture stool samples were used for genomic DNA extraction of the parasite utilizing Gene JET Genomic DNA Purification Kit (Thermo Scientific) following the manufacturer's instructions. DNA of Blastocystis sp. was amplified by PCR assay. Extracted DNA concentrations were assessed, adapted to $5 \mathrm{ng} / \mathrm{ul}$, and were kept at $-20^{\circ} \mathrm{C}$ until processed. A forward primer, RD5 (ATCTGGTTGATC CTGCCAGT) [21], and reverse primer, BhRDr (GAGCTT TT TAACTGCAACAACG) [22] were used.

The primers amplified a 550-585 bp fragment of SSU rDNA sequence of Blastocystis STs. Thermocycler following the PCR cycles and conditions described formerly [21] was performed for Blastocystis-DNA amplification with minor modifications. DNA amplified products were revealed using $1.5 \%$ agarose gel electrophoresis with ultraviolet transillumination after staining by ethidium bromide.

\section{Sequencing and Phylogenetic Analysis of Isolated Blastocystis}

Genome DNA purification kit was used for purification of only 20 PCR products and the primer pair (RD5 and BhRDr) with Big-Dye ${ }^{\circledR}$ Terminator v3.1 was used for sequencing. Ready Reaction Cycle Sequencing Kit (Applied Biosystems, Foster City, CA, USA) also was used following the manufacturer's instructions of the ABI Prism 310 genetic analyzer.
Blastocystis isolates sequences were coincided with reference sequences in the GenBank database. The online BLAST program available at the National Center for Biotechnology Information (NCBI) (http://www.ncbi.nlm.nih. gov/BLAST) was employed. The ClustalW program of the BioEdit software was used to align all sequences [23]. The method of neighbor joining [24] utilizing the Molecular and Evolution Genetic Analysis v7 (MEGA7) software [25] was utilized to create the phylogenetic tree for the sequences.

Evaluation of the phylogenetic tree reliability was done using bootstrapping (1000 replicates). Computing the evolutionary distances was made by the Maximum-Likelihood algorithm with Tamura-3 parameter substitution model using MEGA7.

\section{Statistical Analysis}

Data were collected, tabulated, and coded for statistical analysis using statistical package for social sciences (SPSS) IBM software (version 25), USA. Descriptive analysis of the results in the form of: frequency and percentage for qualitative data and mean \pm standard deviation for quantitative data was calculated. Cross tabulation and Chi-square test $(\chi 2)$ : for comparison between categorical variables and percentage values were done. Calculation of sensitivity, specificity, and positive and negative predictive values was done. $P$ values equal to or less than 0.05 were considered statistically significant.

\section{Results}

This study was conducted on a total of two hundred (200) individuals, ranging between 23 and 79 years, with a mean age of $(48.14 \pm 12.4)$ years. Males were $56 \%$, while females represented $44 \%$ of participants. Blastocystis sp. was identified by culture in $52 \%$ and $42 \%$ of CRC and non-cancer individuals, respectively. Regarding patients categories, the present study revealed non-significant prevalence of Blastocystis infection in CRC group as compared to non-cancer control group $(P=0.101)$.

As shown in Table 1 and Fig. 1, sensitivity in detecting Blastocystis sp. for direct wet preparation and modified trichrome staining was $61.7 \%$ and $100 \%$ and specificity was $72.3 \%$ and $100 \%$, compared to culture on Jones' medium as a golden standard test with diagnostic accuracy rates (82\% and $87 \%$ respectively).

Blastocystis sp. infection were higher in males (53.8\%) than females $(46.2 \%)$, in the $\geq 50-<65$ years age group $(40.4 \%)$ than other age groups in the study and more in rural areas $(76.9 \%)$ than urban areas $(23.1 \%)$. Nevertheless, no statistically associated relation was detected between any of the studied socio-demographic data and 
Table 1 Detection of Blastocystis sp. positive cases among CRC patients and healthy controls $(\mathrm{N}=200)$ using different diagnostic techniques

\begin{tabular}{|c|c|c|c|c|c|c|c|c|c|}
\hline & & \multicolumn{2}{|l|}{ Culture } & \multirow[t]{2}{*}{ Total } & \multicolumn{5}{|c|}{ Accuracy measures } \\
\hline & & $\begin{array}{l}\text { Positive } \\
N=94\end{array}$ & $\begin{array}{l}\text { Negative } \\
N=(106)\end{array}$ & & Variable & $\%$ & OR & $95 \% \mathrm{CI}$ & $P$ value \\
\hline \multirow[t]{2}{*}{ Direct microscopy } & Positive & $\begin{array}{l}58 \\
(61.7)\end{array}$ & $\begin{array}{l}0 \\
(0.00)\end{array}$ & $\begin{array}{l}58 \\
(29.0)\end{array}$ & \multirow{2}{*}{$\begin{array}{l}\text {-Sens } \\
\text {-Spec } \\
\text {-PPV } \\
\text {-NPV } \\
\text {-Acc } \\
\text { - LR+ } \\
\text { - LR- }\end{array}$} & \multirow{2}{*}{$\begin{array}{l}61.7 \\
100 \\
100 \\
74.65 \\
82 \\
61.7 \\
38.29\end{array}$} & \multirow[t]{2}{*}{1.61} & \multirow[t]{2}{*}{$47.3-66.7$} & \multirow[t]{2}{*}{$<0.001 *$} \\
\hline & Negative & $\begin{array}{l}36 \\
(38.3)\end{array}$ & $\begin{array}{l}106 \\
(100.0)\end{array}$ & $\begin{array}{l}142 \\
(71.0)\end{array}$ & & & & & \\
\hline \multirow[t]{2}{*}{ Modified trichrome stain } & Positive & $\begin{array}{l}68 \\
(72.3)\end{array}$ & $\begin{array}{l}0 \\
(0.00)\end{array}$ & $\begin{array}{l}68 \\
(34.0)\end{array}$ & \multirow{3}{*}{$\begin{array}{l}\text {-Sens } \\
- \text { Spec } \\
-P P V \\
-N P V \\
-A c c \\
- \text { LR + } \\
\text { - LR- }\end{array}$} & \multirow{2}{*}{$\begin{array}{l}72.34 \\
100 \\
100 \\
80.30 \\
87 \\
72.3427 .66\end{array}$} & \multirow[t]{2}{*}{2.62} & \multirow[t]{2}{*}{$32.3-75.7$} & \multirow[t]{2}{*}{$<0.001 *$} \\
\hline & Negative & $\begin{array}{l}26 \\
(27.7)\end{array}$ & $106(100.0)$ & $\begin{array}{l}132 \\
(66.0)\end{array}$ & & & & & \\
\hline Total & & $\begin{array}{l}94 \\
(47 \%)\end{array}$ & $106(53 \%)$ & $200(100 \%)$ & & & & & \\
\hline
\end{tabular}

(Sens.): Sensitivity, (Spec.): Specificity, (PPV): Positive predictive value, (NPV): Negative predictive value; (Acc.): Accuracy, (LR+): Positive likelihood ratio, (LR-): Negative likelihood ratio, (OR): Diagnostic odds ratio; (CI): Confidence Interval. *P values $\leq 0.05$ were considered statistically significant

Fig. 1 a Vacuolar forms of Blastocystis sp. (black arrows) in stool sample stained with iodine by $(\times 400)$; $\mathbf{b}$ in vitro cultivation of Blastocystis isolates on Jones' medium after 3 day culture showing the predominance of vacuolar forms by $(\times 400)$

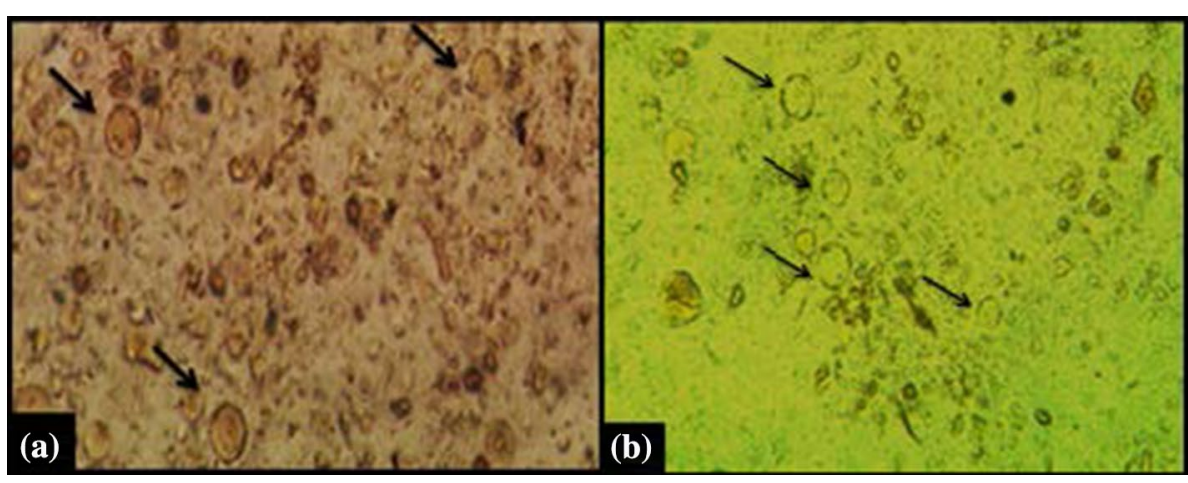

Blastocystis sp. infection among studied CRC patients. Urticaria was the only clinical symptom that showed statistical difference among studied CRC patients with positive Blastocystis sp. infection $(P=0.014)$, as shown in (Table 2).

The studied CRC patients had a significant history of previous surgeries compared to control group, with a high statistically significant value $(P<0.001)$. Other than two patients who had orthopedic surgery, the majority of patients with previous surgical history had surgeries related to colon and gastrointestinal tract diseases (colectomy, piles, hernia, appendectomy, or varicocele ligation). Chronic diseases which include diabetes mellitus (DM) and/or hypertension (HTN) were nearly similar among cases and controls without statistical significance. There was no statistically significant difference detected between Blastocystis sp. infection and CRC patients received radio- and chemotherapy.
Figure 2 demonstrates a significant association between colonoscopy grading of CRC patients and Blastocystis sp. infection. Grade 3 had more positive Blastocystis infection as compared with Grade 2 (65.4 vs. $34.6 \%)$ with a statistically significant difference $(P=0.034)$. Significant association between staging of CRC patients and Blastocystis sp. infection also was identified. With increasing stage, more infection rates were significantly detected in late stage (stage 4) (38.5\%) than stage 3 (34.6\%), and stage 2 (26.9\%) $(P=0.031)$.

Out of 94 cultured Blastocystis (52 cases and 42 control), Blastocystis-DNA was amplified in $44(84.6 \%)$ and $36(85.7 \%)$ of cultured stool from CRC patients and controls, respectively. Four STs (ST1, 2, 3 and 7) of Blastocystis sp. were identified in the study individuals (Table 3). ST1, 2 and 3 were isolated from both cancer and non-cancer individuals; however, ST7 was only isolated from CRC 
Table 2 Association between socio-demographic, past-history data, and different clinical symptoms with Blastocystis infection among studied CRC patients; $(N=100)$ considering in vitro culture as a golden reference test
Fig. 2 Association between Blastocystis sp. infection and both endoscopic grading of CRC $(P=0.034)$ and staging of CRC $(P=0.031)$ showing statistically significant difference

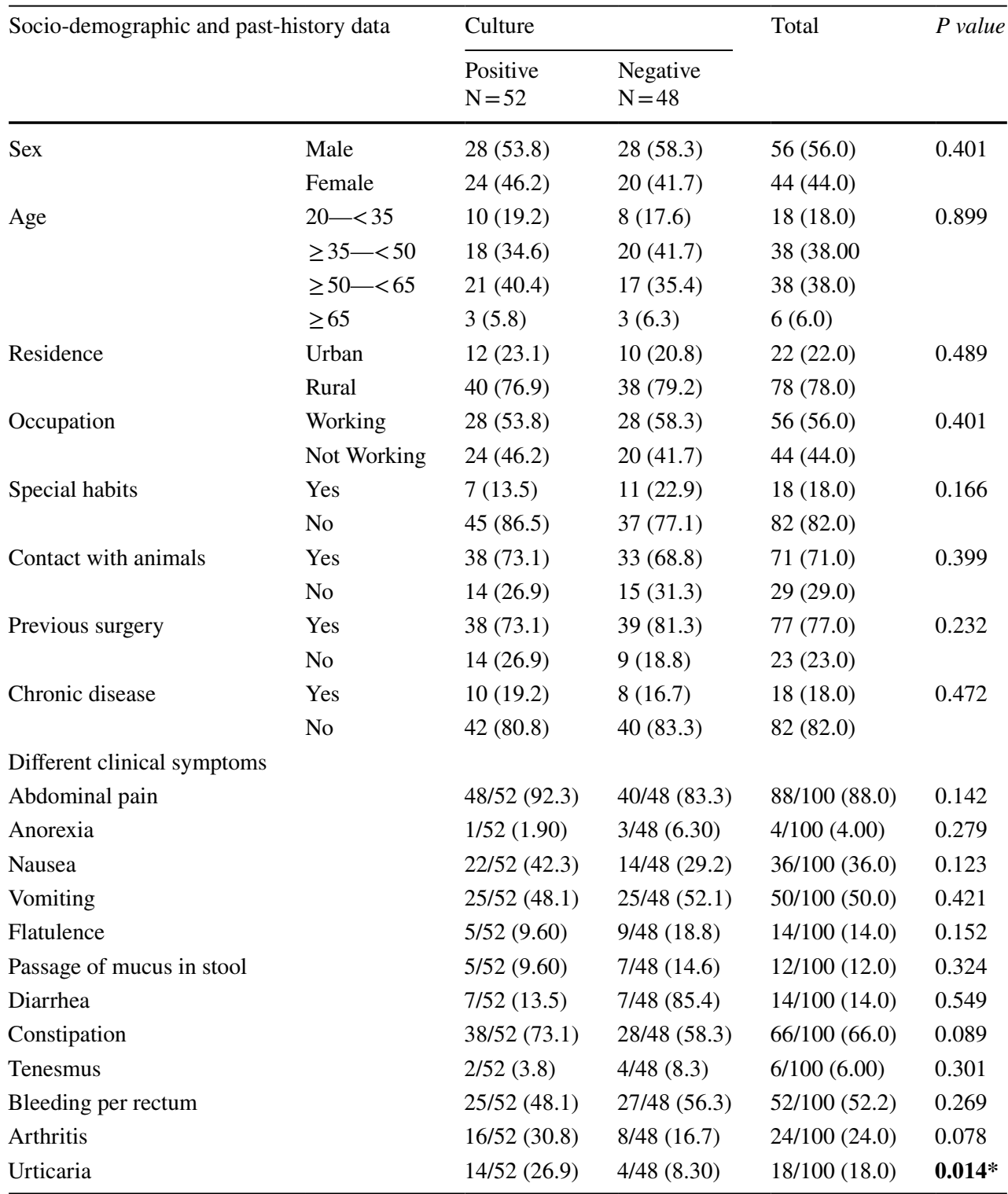

* P values $\leq 0.05$ were considered statistically significant; analysis was carried out using Chi-square test

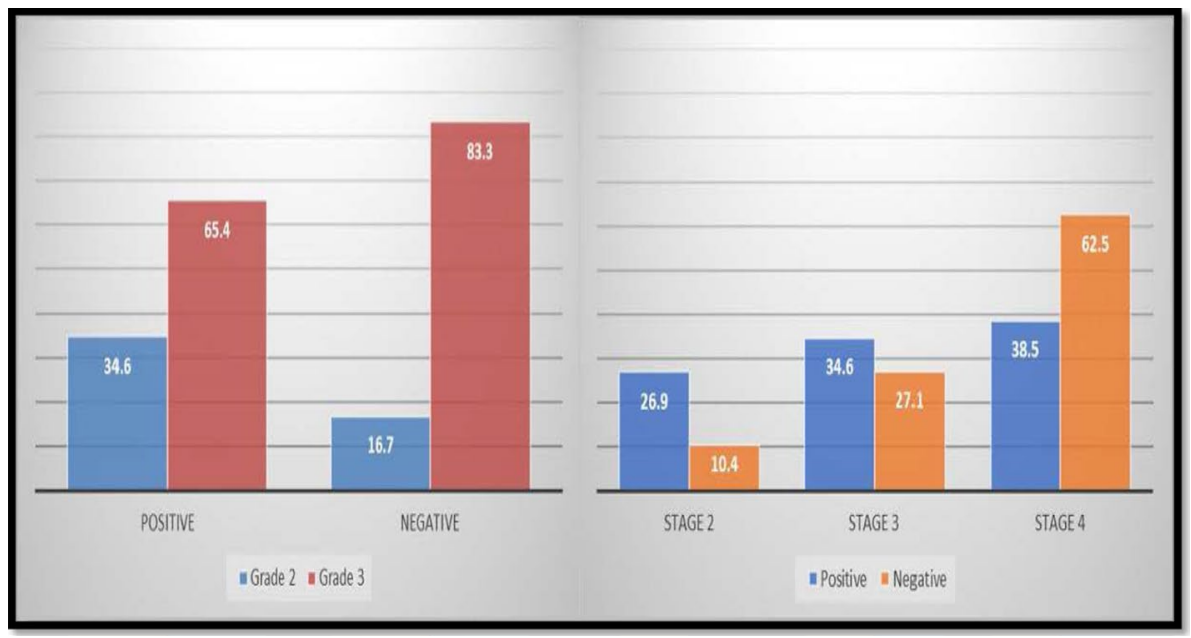


Table 3 Frequency of different Blastocystis STs isolated from CRC patients and control group $(N=20)$

\begin{tabular}{|c|c|c|c|c|}
\hline & \multicolumn{2}{|c|}{$\begin{array}{l}\text { Studied Population } \\
\mathrm{N}(\%)\end{array}$} & \multirow{2}{*}{$\begin{array}{l}\text { Total } \\
\mathrm{N}(\%) \\
20\end{array}$} & \multirow[t]{2}{*}{$P$ value } \\
\hline & $\begin{array}{l}\text { Cases } \\
10\end{array}$ & $\begin{array}{l}\text { Control } \\
10\end{array}$ & & \\
\hline ST1 & $1(10 \%)$ & $4(40 \%)$ & $5(25 \%)$ & 0.142 \\
\hline ST2 & $2(20 \%)$ & $2(20 \%)$ & $4(20 \%)$ & 2.557 \\
\hline ST3 & $4(40 \%)$ & $4(40 \%)$ & $8(40 \%)$ & 0.399 \\
\hline ST7 & $3(30 \%)$ & NO & $3(15 \%)$ & $0.04 *$ \\
\hline
\end{tabular}

${ }^{*} P$ values $\leq 0.05$ were considered statistically significant. Analysis was carried out using Chi-square test

Table 4 Multivariable analysis using logistic regression demonstrates associated risk factors for Blastocystis-infected CRC patients

\begin{tabular}{|c|c|c|c|c|}
\hline & \multirow[t]{2}{*}{ Sig } & \multirow[t]{2}{*}{ OR } & \multicolumn{2}{|c|}{$\begin{array}{l}\text { 95\% C.I. for } \\
\text { (OR) }\end{array}$} \\
\hline & & & Lower & Upper \\
\hline \multicolumn{5}{|l|}{ Clinical Symptoms } \\
\hline Abdominal pain & -0.7370 .474 & 0.478 & 0.063 & 3.607 \\
\hline Anorexia & 1.0980 .660 & 2.999 & 0.023 & 398.451 \\
\hline Nausea & -1.3180 .243 & 0.268 & 0.029 & 2.449 \\
\hline Vomiting & $2.4130 .044 *$ & 11.169 & 1.067 & 116.866 \\
\hline Flatulence & $2.302 \mathbf{0 . 0 3 3 *}$ & 9.997 & 1.203 & 83.101 \\
\hline $\begin{array}{l}\text { Passage of mucus in } \\
\text { stool }\end{array}$ & -0.9100 .478 & 0.403 & 0.033 & 4.981 \\
\hline Diarrhea & -1.9070 .099 & 0.149 & 0.015 & 1.435 \\
\hline Constipation & $-2.816 \mathbf{0 . 0 0 8 *}$ & 0.060 & 0.008 & 0.473 \\
\hline Tenesmus & 1.7350 .383 & 5.670 & 0.115 & 280.672 \\
\hline Bleeding per rectum & 0.6340 .329 & 1.886 & 0.528 & 6.740 \\
\hline Arthritis & -0.7220 .731 & 0.486 & 0.008 & 29.517 \\
\hline Urticaria & -1.5920 .505 & 0.203 & 0.002 & 21.987 \\
\hline \multicolumn{5}{|l|}{ Past history } \\
\hline $\begin{array}{l}\text { Radio- or chemo- } \\
\text { therapy }\end{array}$ & -0.8760 .311 & 0.417 & 0.076 & 2.270 \\
\hline Previous surgery & $2.7300 .012 *$ & 15.333 & 1.819 & 129.244 \\
\hline Chronic disease & -0.6950 .405 & 0.499 & 0.097 & 2.557 \\
\hline \multicolumn{5}{|l|}{ *Colonoscopy } \\
\hline Grade 2 & $0.753 \quad 0.283$ & 2.123 & 0.537 & 8.398 \\
\hline \multicolumn{5}{|l|}{ *Staging } \\
\hline Staging 2 & 1.3620 .172 & 3.904 & 0.553 & 27.537 \\
\hline Staging 3 & $2.820 \mathbf{0 . 0 0 6}^{*}$ & 16.783 & 2.269 & 124.132 \\
\hline Constant & -0.8010 .620 & 0.449 & & \\
\hline
\end{tabular}

Overall model $P$ value $=0.002 *$, Cox \& Snell R Square $=0.329$. CRC grade 2 was compared to grade 3 , CRC stage 2 and 3 were compared to stage 1 , and the whole staging variable was statistically significant with $P$ value $=0.009$ stool samples, with significant association $(P=0.04)$. In CRC patients, ST3 was the most prevalent (40\%), followed by ST7 (30\%), then ST2 (20\%), and ST1 (10\%). While in healthy controls, ST1, ST3, and ST 2 were detected in $(40 \%, 40 \%$, and $20 \%)$, respectively.

Table 4 demonstrates multivariable analysis using logistic regression containing the associated risk factors for Blastocystis-infected CRC patients. Relevant variables entered for prediction were clinical symptoms, past history, colonoscopy, and cancer staging. All prediction variables were categorical; in all variables, the last category was considered as the reference category. In this model, the overall statistically significant value was $(0.002)$.

The presence of vomiting with flatulence in this regression model was considered statistically significant predictor for positive Blastocystis-infected cases $(P=0.044,0.033$ / $\mathrm{OR}=11.169,9.997$, respectively). Constipation was considered a high statistically significant predictor for positive Blastocystis-infected cases $(P=0.003$, OR $=0.060)$. Previous surgery with stage $3 \mathrm{CRC}$ in the current regression model was considered statistically significant predictors for positive Blastocystis-infected cases $(P=0.012,0.006 /$ $\mathrm{OR}=15.333,16.783$, respectively).

Figure 3 illustrates a dendrogram representing neighborjoining phylogenetic tree of Blastocystis STs isolates from CRC patients and non-cancer individuals based on the rDNA gene sequences compared to reference strains (their accession number is presented before their name). Bootstrap analysis was based on 1000 replicates. All obtained sequences were submitted to the GenBank database under accession numbers OL375676-OL375695.

\section{Discussion}

The annual incidence of CRC worldwide is over 1 million in men and nearly 79500 in women, with 475000 mortalities in men and 387000 in women [26]. The prevalence of Blastocystis infection in the current study is 52\% in CRC patients and $42 \%$ in control group considering in vitro culture as a golden reference test. Although this difference is statistically non-significant, it agrees with findings from previous studies [1, 11, 27]. Mohamed et al. [1] reported a significant high prevalence of Blastocystis in CRC patients as compared to control population. In Uzbekistan, higher prevalence (80\%) of Blastocystis sp. infection was detected in CRC patients [11]. Lower prevalence of blastocystosis was detected in Saudi Arabia CRC patients $(29.7 \%$ and 26.6\%) [1, 27]. These findings confirm the association between Blastocystis sp. and CRC patients, and assure that Blastocystis infection is common and should be routinely screened for immunocompromised patients. 
Fig. 3 Dendrogram representing neighbor-joining phylogenetic tree of Blastocystis STs isolates from CRC patients and non-cancer individuals based on the rDNA gene sequences compared to reference strains (their accession number is presented before their name). Bootstrap analysis was based on 1000 replicates

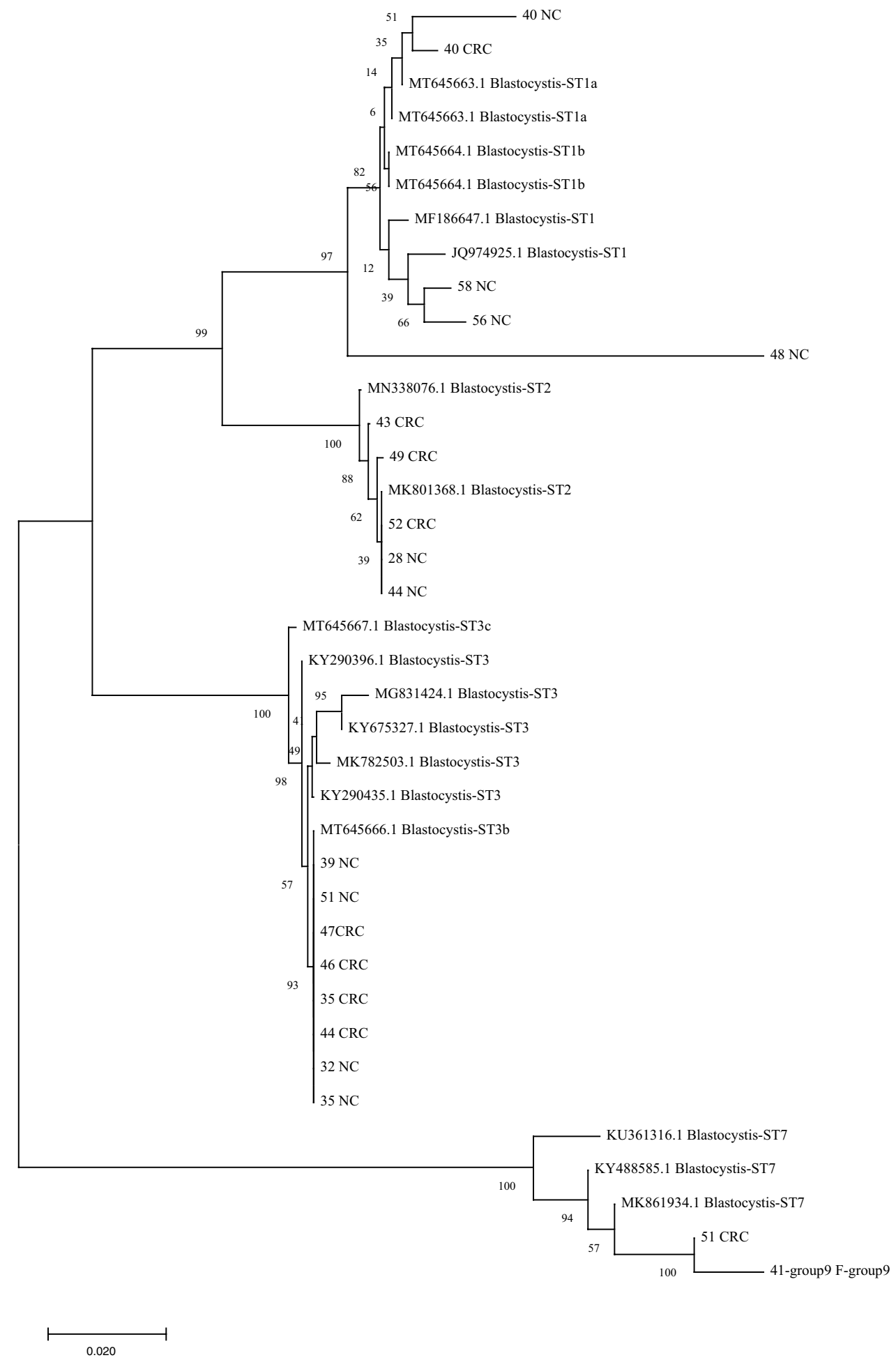

Blastocystis sp. was detected in the current study in 30 $(30 \%), 36(36 \%)$, and $52(52 \%)$ by wet mount preparation, modified trichrome staining and culture, respectively, in stool specimens of CRC patients. Culture on Jones' medium detected the highest number of Blastocystis sp. in human fecal samples, in agreement with the results of previous studies [17, 28-30]. Similarly, a detection rate of in vitro culture of $53 \%, 53.6 \%$, and $51.3 \%$ were reported in previous studies [28, 31, 32]. On the other hand, other studies reported that PCR assay had the highest percentage of Blastocystis sp. detection [14, 33, 34]. Khalifa [35] reported that modified trichrome stain was considered to be the best stain 
to identify Blastocystis. In the present study, modified trichrome stain detected $72 \%$ of total cases detected by culturing with better quality of staining, but it has the disadvantage of being time-consuming and expensive.

Blastocystis sp. infection in CRC patients was higher in males (53.8\%) than females (46.2\%), and this result was non-significant but in agreement with results which reported that Blastocystis frequency was higher in males than females (19\% vs. 6.5\%) among cancer patients [36] and showed that $B$. hominis infection was more frequent in male than in female CRC patients [27]. These findings may be attributed to more outdoor activities and exposure to infection sources in male patients. Moreover, it was reported that the overall incidence of bowel cancer is higher in males than in females [37, 38]. On the other hand, some investigators reported an equal ratio of Blastocystis sp. infection for males and females cancer patients [39].

Blastocystis infection was higher for the age group 50-65 years $(40.4 \%)$ without statistically significant difference. Similarly, a previous study has found that the Blastocystis sp. infection was more prominent in the older age group with mean age of 57 years [40]. Meanwhile, Blastocystis sp. was more common in age group of 45-65 years $[36,41]$. In contrast, others concluded that Blastocystis sp. infection was common in 15-30 years age group [42,43]. However, other studies recorded that Blastocystis sp. infection was common in individuals younger than 15 [44, 45]. This variation in the results regarding age may be explained by the variability of the number and category of patients participated in each study.

Patients living in rural areas had a higher rate of Blastocystis infection than urban areas $(76.9 \%$ vs $23.1 \%)$ without statistical significance. This finding was in agreement with other researchers $[17,28]$. This can be due to frequent contact with animals and soil, low sanitation level, large family size, and unhealthy drinking water sources [28]. This parasitic infection could be an index to the level of public health [46]. On the other side, Blastocystis sp. infection was more common in urban than in rural habitants [27].

Presence of vomiting and flatulence in the current regression model was considered statistically significant predictor for positive Blastocystis infection in CRC patients, which coincides with Hamdy et al. [28] who demonstrated that only vomiting and anorexia showed significant association with blastocystosis. Similarly, the previous studies reported that only flatulence showed statistical significance with blastocystosis [17, 47].

Constipation was also considered a statistically significant predictor for positive Blastocystis infection, a finding that may be attributed to the prevalence of constipation in CRC patients [48].

The most frequent symptom was abdominal pain in $92.3 \%$ of the studied CRC patients, followed by constipation in
$73.1 \%$ of cases. The least frequent symptoms in studied CRC patients were anorexia in (1.9\%) and tenesmus in (3.8\%). All other clinical symptoms except urticaria $(P=0.014)$ showed non-statistically significant differences $(P>0.05)$. This result agrees with another study where there was no significant difference in gastrointestinal symptoms of Blastocystis-infected cancer patients [14].

Pathogenesis of urticaria can be attributed to involvement of multiple protozoan parasites [49]. Blastocystis is one of the most encountered enteric parasites in asymptomatic and symptomatic individuals [50]. Previous research correlates Blastocystis infection with skin disorders and acute or chronic urticaria [51-55]. The mechanism by which Blastocystis $\mathrm{sp}$. induces dermal lesions is still not clear; certain immune responses or some alterations of gut microbiota may be incriminated [56-58]. Many studies associate cutaneous pathology with the amoeboid form of Blastocystis [52, 53]. Furthermore, it has been found that Blastocystis eradication results in complete resolution of dermal lesions which may indicate the role of Blastocystis in the skin disorders. However, despite prolonged antimicrobial therapy, recurrence of symptoms has been documented [59].

In the current study, CRC patients had history of previous surgeries $(77 \%)$ compared to controls $(10 \%)$ with a statistically significant difference. Previous surgery was considered a statistically significant predictor for Blastocystis infection. This contradicts a previous study which found that the entire number of CRC patients infected with Blastocystis sp. was the same after surgery and excision of tumors did not significantly reflect on the intensity or frequency of Blastocystis sp. infection [11].

In the present study, Blastocystis sp. infection was frequently detected among CRC patients who had received radio or chemotherapy without statistical significance. Two studies showed that Blastocystis was more frequent in patients who had received eight or more cycles of chemotherapy than those had received less or none cycles of chemotherapy $[14,36]$. On the contrary, Toychiev et al., [11] reported that the number of Blastocystis-infected CRC patients after chemotherapy was constant $(75 \%)$ and showed significant difference as compared to control group [11].

It has been documented that $B$. hominis has a pathogenic role in CRC due to induction of colonic epithelial cell mutation in addition to other factors, such as genetic predisposition, may trigger CRC [60]. The possible carcinogenic role of Blastocystis infection in humans, especially in CRC patients, has been previously discussed [61, 62].

The present study demonstrates a significant association between colonoscopy grading of CRC patients and Blastocystis infection. Grade 3 had more positive Blastocystis infection as compared with Grade $2(65.4$ vs. $34.6 \%)$ with a statistically significant difference $(P=0.034)$. Our results agree with others which demonstrate a notable inflammatory 
cellular infiltration and greater presence of mucin in grade 3 CRC patients infected with Blastocystis [27]. Kumarasamy et al. suggested that Blastocystis sp. enhanced Azoxymethane induced carcinogenesis by promoting oxidative damage to the intestinal epithelium in Blastocystis sp. infected rats [5].

There is a significant association between staging of CRC patients and Blastocystis infection. With each increasing tumor stage, more Blastocystis infection rates were detected, $38.5 \%, 34.6 \%$, and $26.9 \%$ in stages 4,3 , and 2 , respectively $(P=0.031)$. Moreover, stage $3 \mathrm{CRC}$ in the multivariate regression model was considered a statistically significant predictor for positive Blastocystis infection. This was reported by previous researches which suggest that the infection may promote growth of CRC cells [11, 62]. Blastocystis infection was detected in all the cancer stages in a previous study; however, it showed higher positivity in stages 3 and 4 [27]. On the other hand, the prevalence $B$. hominis infection in CRC patients of each stage had no statistical significance among different stages [60].

Our findings support the hypothesis that the infection with Blastocystis sp. has a potential role in the pathogenesis of CRC and this is supported by several studies [1, 11, 14, 27]. The pathophysiological mechanism of Blastocystis sp. infections in CRC patients is attributed to production of multiple immunological components of inflammatory cells, as well as a result of the downregulation of the inflammatory cytokines and host immune response in the early stages of CRC, which improve its survival [27].

B. hominis infections can initiate free radical formation, such as superoxide anion and nitric oxide to CRC; however, its chronic infections lead to multiple pathophysiological disorders involving mutations with long-lasting oxidative stress causing cancer [63, 64]. Nevertheless, another study suggested that blastocystosis does not stimulate the colonic tissue to form a tumor, but it may intensify an established colorectal tumor [65]. There is a higher marked degree of antioxidant processes and intensification of the oxidative protein damage associated with Blastocystis infection [66].

Since PCR is known as an expensive, laborious technique and inefficient tool in screening diagnostic targets for large of sample sizes [67], in the current study, culture was more sensitive than PCR, PCR was positive for $85 \%$ of the 94 individuals that were positive for Blastocystis using culture, which may be attributed to the disintegration of the parasite DNA during storage process or the inability of STs primer to detect certain STs (majority of ST4, ST8, and ST9) [43, 68]. In earlier literature, Blastocystis ST1, 2, and 3 were isolated from both cancer and non-cancer individuals; however, ST7 was only isolated from CRC stool samples, with significant association. In the current study, for CRC patients, ST3 was most prevalent (40\%), followed by ST7 (30\%) which was found in CRC patients for the first time, ST2 (20\%), and ST1
(10\%). The obtained results of the present study were similar to others which reported that ST3 was the most prevalent subtype in Iranian cancer patients (37.5\%), followed by ST2 (33.3\%), ST1 (20.9\%), and ST7 (8.3\%) in two patients with hematological and cranial malignancies, with ST3 was the only subtype found in CRC patients in their study [14], while ST3 was the most abundant subtype in Turkey and Malaysia in cancer patients [36, 69-71].

In this study, there is no difference in the distribution of ST3 for CRC patients and control group. Research has indicated that ST3 is more anthropogenic; the most frequently isolated subtype in humans, and may be present in symptomatic and asymptomatic individuals [72-74]. ST1 was the most common subtype for Saudi Arabia CRC patients, followed by ST2 and ST5, respectively [1]. Moreover, a strong association risk was demonstrated between Blastocystis ST1 and CRC which confirmed the carcinogenic postulated effect of certain Blastocystis STs and their probable reflection on CRC [1]. For CRC patients, it was reported that ST1 was the most common subtype in Iraq followed by ST3 [40]. Similarly, in China, ST1 was more prevalent than ST3 in cancer patients with diarrhea [41].

Interestingly, our study revealed the rare subtype (ST7) in CRC patients. The three patients having ST7 were males, came from urban areas, were 54, 51, and 53 years old, had colonic adenocarcinoma grade 2 , and previous history of colectomy. This subtype, as the ST5, ST6, and ST8, is rarely found in humans, and it has previously been proposed that these are rare zoonotic subtypes detected in humans [75]. Also, ST7 has been previously recognized in farm animals, supporting its zoonotic transmission through handling of animal [76]. Moreover, ST7 has potentially caused gut microbiota imbalance and decreased the level of beneficial gut bacteria like Lactobacillus and Bifidobacterium [77, 78]. ST7 has been detected in humans in many countries, with prevalence rates extending from $0.8 \%$ in Nigeria [79] to $17.9 \%$ in Thailand [80]. A recent study in Singapore has identified ST7 as the predominant subtype in diarrheal patients [81].

\section{Conclusion}

Blastocystosis plays an essential role in the CRC morbidity. It is highly prevalent in CRC patients, predominantly in the latest grades and stages of CRC. Blastocystis ST1, 2 , and 3 were isolated from both cancer and non-cancer individuals; however, the rare pathogenic ST7 was isolated for first time in CRC stool samples, with significant association. Cancer growth and progress is highly correlated with gut inflammatory microenvironment; consequently, CRC patients require screening for Blastocystis 
sp. infection. Although the substantial role of intestinal microbiota is accepted as a significant component in promoting and development of CRC, studies evaluating the parasitic biology of the gut microbiota are still limited, with contradictory findings. To determine whether certain STs of Blastocystis are associated with CRC, or are actual carcinogens would require further research.

Authors' Contribution All manuscript authors contributed to every aspect of it; idea of the research, study design, collection of materials, methodology, writing the paper, and revising/editing it. All authors read and approved the final manuscript.

Availability of Data and Materials Not applicable.

Code Availability Not applicable.

\section{Declarations}

Conflict of Interest The authors have no relevant financial or non-financial interests to disclose.

Ethics Approval This study was performed in line with the principles of the Declaration of Helsinki. Approval was granted by the Ethics Committee of Beni-Suef University, Faculty of Medicine (No. FWA00015574).

Consent to Participate Informed consent was obtained from all individual participants included in the study. The aim of the study was explained to all included participants. Participants who had positive results were personally informed or through their physicians to receive proper medications.

Consent for Publication Not applicable.

\section{References}

1. Mohamed AM, Ahmed MA, Ahmed SA, Al-Semany SA, Alghamdi SS, Zaglool DA (2017) Predominance and association risk of Blastocystis hominis subtype I in colorectal cancer: a case control study. Infect Agent Cancer 12(1):21. https://doi.org/10. 1186/s13027-017-0131-z

2. Potes-Morales C, Osorio-Delgado LA, Carranza JC, Vallejo GA (2020) The first molecular detection of Blastocystis subtypes in human faecal samples from Ibague. Colombia. Parasite Epidemiol Control 9:e00132. https://doi.org/10.1016/j.parepi.2020.e00132

3. Zanetti ADS, Malheiros AF, de Matos TA, Longhi FG, Moreira LM, Silva SL, et al (2020) Prevalence of Blastocystis sp. infection in several hosts in Brazil: a systematic review and metaanalysis. Parasit Vectors 13(1): 30. https://doi.org/10.1186/ s13071-020-3900-2

4. Gong B, Liu X, Wu Y, Xu N, Xu M, Yang F et al (2019) Prevalence and subtype distribution of Blastocystis in ethnic minority groups on both sides of the China-Myanmar border, and assessment of risk factors. Parasite 26:46. https://doi.org/10.1051/paras ite/2019046

5. Kumarasamy V, Kuppusamy UR, Jayalakshmi P, Samudi C, Ragavan ND, Kumar S (2017) Exacerbation of colon carcinogenesis by Blastocystis sp. PLoS ONE 12(8):e0183097. https://doi.org/ 10.1371/journal.pone.0183097

6. Deng L, Chai Y, Zhou Z, Liu H, Zhong Z, Hu Y et al (2019) Epidemiology of Blastocystis sp. infection in China: a systematic review. Parasite 26:41. https://doi.org/10.1051/parasite/2019042

7. Padukone S, Mandal J, Parija SC (2017) Severe Blastocystis subtype 3 infection in a patient with colorectal cancer. Trop Parasitol 7(2):122-124. https://doi.org/10.4103/tp.TP_87_15

8. Ferlay J, Colombet M, Soerjomataram I, Mathers C, Parkin D, Piñeros, M, et al (2018) Estimating the global cancer incidence and mortality in 2018: GLOBOCAN sources and methods. Int $\mathbf{J}$ Cancer 144(8): 1941-1953. https://doi.org/10.1002/ijc.31937

9. Rawla P, Sunkara T, Barsouk A (2019) Epidemiology of colorectal cancer: incidence, mortality, survival, and risk factors. Prz Gastroenterol 14(2):89-103. https://doi.org/10.5114/pg.2018.81072

10. Rositch AF (2020) Global burden of cancer attributable to infections: the critical role of implementation science. Lancet Glob Health 8(2):e153-e154. https://doi.org/10.1016/S2214-109X(20) 30001-2

11. Toychiev A, Abdujapparov S, Imamov A, Navruzov B, Davis $\mathrm{N}$, Badalova N, et al (2018) Intestinal helminths and protozoan infections in patients with colorectal cancer: prevalence and possible association with cancer pathogenesis. Parasitol Res117 (12): 3715-3723. https://doi.org/10.1007/s00436-018-6070-9

12. Alfellani MA, Taner-Mulla D, Jacob AS, Imeede CA, Yoshikawa H, Stensvold CR et al (2013) Genetic diversity of Blastocystis in livestock and zoo animals. Protist 164(4):497-509. https://doi.org/ 10.1016/j.protis.2013.05.003

13. Ramírez JD, Sánchez A, Hernández C, Flórez C, Bernal MC, Giraldo JC et al (2016) Geographic distribution of human Blastocystis subtypes in South America. Infect Genet Evol 41:32-35. https://doi.org/10.1016/j.meegid.2016.03.017

14. Asghari A, Zare M., Hatam G, Shahabi S, Gholizadeh F, Motazedian M (2020) Molecular identification and subtypes distribution of Blastocystis sp. isolated from children and adolescent with cancer in Iran: evaluation of possible risk factors and clinical features. Acta Parasitol 65(2): 462-473. https://doi.org/10.2478/ s11686-020-00186-2

15. American Society of Clinical Oncology. Colorectal Cancer. 2017: https://www.cancer.net/cancer-types/colorectal-cancer/view-all

16. Garcia LS (2007) Diagnostic medical parasitology, 5th edn. ASM Press, Washington, pp 759-763

17. El-Badry AA, El Wahab WMA, Hamdy DA, Aboud A (2018) Blastocystis subtypes isolated from irritable bowel syndrome patients and co-infection with Helicobacter pylori. Parasitol Res 117(1):127-137. https://doi.org/10.1007/s00436-017-5679-4

18. Jones W (1946) The experimental infection of rats with Entamoeba histolytica; with a method for evaluating the anti-amoebic properties of new compounds. Ann Trop Med Parasitol 40(2):130-140. https://doi.org/10.1080/00034983.1946.11685270

19. Zaman V, Khan K (1994) A comparison of direct microscopy with culture for the diagnosis of Blastocystis hominis. Southeast Asian J Trop Hyg Public Health 25:792-793

20. Abdulsalam AM, Ithoi I, Al-Mekhlafi HM, Al-Mekhlafi AM, Ahmed A, Surin J (2013) Subtype distribution of Blastocystis isolates in Sebha. Libya. PLoS One 8(12):e84372. https://doi.org/ 10.1371/journal.pone.0084372

21. Clark CG (1997) Extensive genetic diversity in Blastocystis hominis. Mol Biochem Parasitol 87(1):79-83. https://doi.org/10.1016/ S0166-6851(97)00046-7

22. Scicluna SM, Tawari B, Clark CG (2006) DNA barcoding of Blastocystis. Protist 157(1):77-85. https://doi.org/10.1016/j.protis. 2005.12.001

23. Hall TA (1999) BioEdit: a user-friendly biological sequence alignment editor and analysis program for Windows 95/98/NT. Nucl 
Acids Symp Ser 41:95-98. https://doi.org/10.14601/PHYTO PATHOL_MEDITERR-14998U1.29

24. Saitou N, Nei M (1987) The neighbor-joining method: a new method for reconstructing phylogenetic trees. Mol Biol Evol 4(4):406-425. https://doi.org/10.1093/oxfordjournals.molbev. a040454

25. Kumar S, Stecher G, Tamura K (2016) MEGA7: Molecular Evolutionary Genetics Analysis version 7.0 for bigger datasets. Mol Biol Evol 33(7):1870-1874. https://doi.org/10.1093/molbev/ msw054

26. Allemani C, Matsuda T, Di Carlo V, Harewood R, Matz M, Nikšić $M$ et al (2018) Global surveillance of trends in cancer survival 2000-14 (CONCORD-3): analysis of individual records for 37 513025 patients diagnosed with one of 18 cancers from 322 population-based registries in 71 countries. Lancet 391(10125):10231075. https://doi.org/10.1016/S0140-6736(17)33326-3

27. Hawash YA, Ismail KA, Saber T, Eed EM, Khalifa AS, Alsharif KF et al (2020) Predominance of Infection With Blastocystis hominis in Patients With Colorectal Cancer and Its Association With High Mucin Content, Infiltration of Inflammatory Cells and Elevated Serum Tumor Necrosis Factor $\alpha$. Infect Dis Clin Pract 29(1):e32-e38. https://doi.org/10.1097/IPC.0000000000000931

28. Hamdy DA, Abd El Wahab WM, Senosy SA, Mabrouk AG (2020) Blastocystis sp. and Giardia intestinalis co-infection profile in children suffering from acute diarrhea. J Parasit Dis 44(1): 88-98. https://doi.org/10.1007/s12639-019-01165-9

29. Santos HJ, Rivera WL (2013) Comparison of direct fecal smear microscopy, culture, and polymerase chain reaction for the detection of Blastocystis sp. in human stool samples. Asian Pac J Trop Med 6(10):780-784. https://doi.org/10.1016/S1995-7645(13) 60138-8

30. Popruk S, Pintong A, Radomyos P (2013) Diversity of Blastocystis subtypes in humans. J Trop Med Parasitol 36:88-97

31. EL-Marhoumy S M, EL-Nouby KA, Shoheib ZS, Salama AM, (2015) Prevalence and diagnostic approach for a neglected protozoon Blastocystis hominis. Asian Pac J Trop Dis 1:51-59. https:// doi.org/10.1016/S2222-1808(14)60626-5

32. Salazar-Sánchez RS, Ascuña-Durand K, Ballón-Echegaray J, Vásquez-Huerta V, Martínez-Barrios E, Castillo-Neyra R (2020) Socio-demographic determinants associated with Blastocystis infection in Arequipa. Peru. Am J Trop Med Hyg 104(2):700-707. https://doi.org/10.4269/ajtmh.20-0631

33. Tan KS (2008) New insights on classifcation, identifcation, and clinical relevance of Blastocystis spp. Clin Microbiol Rev 21(639665):367. https://doi.org/10.1128/CMR.00022-0827

34. Parkar U, Traub RJ, Kumar S, Mungthin M, Vitali S, Leelayoova S, Morris K, Thompson RC (2007) Direct characterization of Blastocystis from faeces by PCR and evidence of zoonotic potential. Parasitology 134:359-367. https://doi.org/10.1017/S0031 182006001582

35. Khalifa AM (1999) Diagnosis of Blastocystis hominis by different staining techniques. J Egypt Soc Parasitol 29(1):157-165

36. Yersal O, Malatyali E, Ertabaklar H, Oktay E, Barutca S, Ertug S (2016) Blastocystis subtypes in cancer patients: analysis of possible risk factors and clinical characteristics. Parasitol Int 65(6):792-796. https://doi.org/10.1016/j.parint.2016.02.010

37. Cook MB, Dawsey SM, Freedman ND, Inskip PD, Wichner SM, Quraishi SM et al (2009) Sex disparities in cancer incidence by period and age. Cancer Epidemiol Biomarkers Prev 18(4):11741182. https://doi.org/10.1158/1055-9965.EPI-08-1118

38. Murphy G, Devesa SS, Cross AJ, Inskip PD, McGlynn KA, Cook MB (2011) Sex disparities in colorectal cancer incidence by anatomic subsite, race and age. Int J Cancer 128(7):1668-1675. https://doi.org/10.1002/ijc.25481
39. Mohammad NA, Al-Mekhlafi HM, Moktar N, Anuar TS (2017) Prevalence and risk factors of Blastocystis infection among underprivileged communities in rural Malaysia. Asian Pac J Trop Med 10(5):491-497. https://doi.org/10.1016/j.apjtm.2017.05.001

40. Hammood AM, Ahmed BA, SalmanY J (2016) Blastocystis hominis detection among gastrointestinal disorders' patients in Kirkuk Province using three different laboratory methods. Int J Curr Microbiol App Sci. 5: 883-901. https://doi.org/10.20546/ ijcmas.2016.507.101

41. Zhang W, Ren G, Zhao W, Yang Z, Shen Y, Sun Y et al (2017) Genotyping of Enterocytozoon bieneusi and subtyping of Blastocystis in cancer patients: relationship to diarrhea and assessment of zoonotic transmission. Front Microbiol 8:1835. https://doi.org/ 10.3389/fmicb.2017.01835

42. Dagci H, Özgür K, Demirel M, Mandiracioglu A, Aydemir S, Ulas S et al (2014) Epidemiological and diagnostic features of Blastocystis infection in symptomatic patients in Izmir province. Turkey Iran J Parasitol 9(4):519

43. Gabr N S, Latif M ZA, Mohamed RM, Belal US, Sanadeki MM, Ghany WMA (2018) Blastocystis sp. infection among IBS patients: various diagnostic methods and epidemiological study. JESP 48(1): 119-128. https://doi.org/10.21608/JESP.2018.77474

44. Nascimento SA, Moitinho Md LR (2005) Blastocystis hominis and other intestinal parasites in a community of Pitanga City, Paraná State, Brazil. Rev Inst Med Trop Sao Paulo 47(4):213-217. https://doi.org/10.1590/s0036-46652005000400007

45. Zabolinejad N, Berenji F, Bayati Eshkaftaki E, Badeii Z, Banihashem A, Afzalaqaei M (2013) Intestinal parasites in children with lymphohematopoietic malignancy in Iran. Mashhad Jundishapur Journal of Microbiology 6(6):e7765. https://doi.org/10. 5812/jjm. 7765

46. Javanmard E, Niyyati M, Ghasemi E, Mirjalali H, Aghdaei HA, Zali MR (2018) Impacts of human development index and climate conditions on prevalence of Blastocystis: a systematic review and meta-analysis. Acta Trop 185:193-203. https://doi.org/10.1016/j. actatropica.2018.05.014

47. Ramirez-Miranda ME, Hernandez-Castellanos R, LopezEscamilla E, Moncada D, Rodriguez-Magallan A, Pagaza-Melero $\mathrm{C}$ et al (2010) Parasites in Mexican patients with irritable bowel syndrome: a case-control study. Parasit Vectors 3(1):96. https:// doi.org/10.1186/1756-3305-3-96

48. Mjoli M, Govindasamy V, Madiba TE (2017) What is the diagnostic yield of colonoscopy in patients with a referral diagnosis of constipation in South Africa? S Afr J Surg 55(3):14-18

49. Kolkhir P, Balakirski G, Merk HF, Olisova O, Maurer M (2016) Chronic spontaneous urticaria and internal parasites - a systematic review. Allergy 71(3):308-322. https://doi.org/10.1111/all.12818

50. Bahrami F, Babaei E, Badirzadeh A, Riabi TR, Abdoli A (2019) Blastocystis, urticaria, and skin disorders: review of the current evidences. Eur J Clin Microbiol Infect Dis. https://doi.org/10. 1007/s10096-019-03793-8

51. Vogelberg C, Stensvold C R, Monecke S, Ditzen A, Stopsack K, Heinrich-Gräfe U et al (2010) Blastocystis sp. subtype 2 detection during recurrence of gastrointestinal and urticarial symptoms. Parasitol Int 59(3): 469-471. https://doi.org/10.1016/j.parint.2010.03. 009

52. Hameed DMA, Hassanin OM, Zuel-Fakkar NM (2011) Association of Blastocystis hominis genetic subtypes with urticaria. Parasitol Res 108(3):553-560. https://doi.org/10.1007/ s00436-010-2097-2

53. Zuel-Fakkar N, Abdel Hameed D, Hassanin O (2011) Study of Blastocystis hominis isolates in urticaria: a case-control study. Clin Exp Dermatol 36(8):908-910. https://doi.org/10.1111/j. 1365-2230.2011.04127.x 
54. Verma R, Delfanian K (2013) Blastocystis hominis associated acute urticaria. Am J Med Sci 346(1):80-81. https://doi.org/10. 1097/MAJ.0b013e3182801478

55. Lepczyńska M, Chen WC, Dzika E (2016) Mysterious chronic urticaria caused by Blastocystis sp.? Int J Dermatol 55(3):259266. https://doi.org/10.1111/ijd.13064

56. Roberts T, Stark D, Harkness J, Ellis J (2014) Update on the pathogenic potential and treatment options for Blastocystis sp. Gut Pathog 6(1): 17. https:// doi: https://doi.org/10.1186/ 1757-4749-6-17

57. Ajjampur SS, Tan KS (2016) Pathogenic mechanisms in Blastocystis sp.-Interpreting results from in vitro and in vivo studies. Parasitol Int 65(6): 772-779. https://doi.org/10.1016/j.parint. 2016.05.007

58. Stensvold CR, van der Giezen M (2018) Associations between gut microbiota and common luminal intestinal parasites. Trends Parasitol 34(5):369-377. https://doi.org/10.1016/j.pt.2018.02.004

59. Mirza H, Tan KSW (2012) Clinical Aspects of Blastocystis Infections: Advancements Amidst Controversies. In H. Mehlhorn, K. S. W. Tan \& H. Yoshikawa (Eds.), Blastocystis: Pathogen or Passenger? An Evaluation of 101 Years of Research (Vol. 4, pp. 65-84): Springer Berlin Heidelberg.

60. Steer H (2007) Blastocystis hominis and colorectal cancer. Ann R Coll Surg Engl 89(5):539. https://doi.org/10.1308/003588407X 202155

61. Chandramathi S, Suresh K, Kuppusamy UR (2010) Solubilized antigen of Blastocystis hominis facilitates the growth of human colorectal cancer cells, HCT116. Parasitol Res 106(4):941-945. https://doi.org/10.1007/s00436-010-1764-7

62. Chan KH, Chandramathi S, Suresh K, Chua KH, Kuppusamy UR (2012) Effects of symptomatic and asymptomatic isolates of Blastocystis hominis on colorectal cancer cell line, HCT116. Parasitol Res 110(6):2475-2480. https://doi.org/10.1007/ s00436-011-2788-3

63. Chandramathi S, Suresh K, Anita ZB, Kuppusamy UR (2012) Infections of Blastocystis hominis and microsporidia in cancer patients: are they opportunistic? Trans R Soc Trop Med Hyg 106(4):267-269. https://doi.org/10.1016/j.trstmh.2011.12.008

64. Reuter S, Gupta SC, Chaturvedi MM, Aggarwal BB (2010) Oxidative stress, inflammation, and cancer: how are they linked? Free Radic Biol Med 49(11):1603-1616. https://doi.org/10.1016/j.freer adbiomed.2010.09.006

65. Kumarasamy V, Kuppusamy UR, Samudi C, Kumar S (2013) Blastocystis sp subtype 3 triggers higher proliferation of human colorectal cancer cells, HCT116. Parasitol Res 112(10):35513555. https://doi.org/10.1007/s00436-013-3538-5

66. EL-Khazragy N, Saad GA (2019) Blastocystis sp. enhances oxidative stress-induced carcinogenesis in colorectal cancer: in vivo experimental study. IJAR 7(9): 1231-1238. https://doi.org/10. 21474/IJAR01/9777

67. Haque R, Roy S, Siddique A, Mondal U, Rahman SM, Mondal D et al (2007) Multiplex real-time PCR assay for detection of Entamoeba histolytica, Giardia intestinalis, and Cryptosporidium sp. Am J Trop Med Hyg 76(4):713-717. https://doi.org/10.4269/ ajtmh.2007.76.713

68. Stensvold CR (2013) Blastocystis: genetic diversity and molecular methods for diagnosis and epidemiology. Trop Parasitol 3(1):26. https://doi.org/10.4103/2229-5070.113896
69. Tan T, Ong S, Suresh K (2009) Genetic variability of Blastocystis sp. isolates obtained from cancer and HIV/AIDS patients. Parasitol Res 105(5):1283-1286. https://doi.org/10.1007/ s00436-009-1551-5

70. Mülayim S, Aykur M, Dağcı H, Dalkılıç S, Aksoy A, Kaplan M (2021) Investigation of Isolated Blastocystis Subtypes from Cancer Patients in Turkey. Acta Parasitol. https://doi.org/10.1007/ s11686-020-00322-y

71. Kumarasamy V, Roslani A C, Rani KU, Govind SK (2014) Advantage of using colonic washouts for Blastocystis detection in colorectal cancer patients. Parasit Vectors 7(1):162. https://doi.org/10. 1186/1756-3305-7-162

72. Clark CG, van der Giezen M, Alfellani MA, Stensvold CR (2013) Recent developments in Blastocystis research. Adv Parasitol 82: 1-32. https://doi.org/10.1016/B978-0-12-407706-5.00001-0

73. Ragavan N D, Govind SK, Chye TT, Mahadeva S (2014) Phenotypic variation in Blastocystis sp. ST3. Parasit Vectors 7(1): 1-10. https://doi.org/10.1186/1756-3305-7-404.

74. Cakir F, Cicek M, Yildirim IH (2019) Determination the subtypes of Blastocystis sp. and evaluate the effect of these subtypes on pathogenicity. Acta Parasitol 64(1): 7-12. https://doi.org/10.2478/ s11686-018-00002-y

75. Stensvold CR, Clark CG (2016) Current status of Blastocystis: a personal view. Parasitol Int 65(6): 763-771. https://doi.org/10. 1016/j.parint.2016.05.015

76. Gabrielli S, Palomba M, Furzi F, Brianti E, Gaglio G, Napoli E, Rinaldi L, Alburqueque RA, Mattiucci S (2021) Molecular Subtyping of Blastocystis sp. Isolated from Farmed Animals in Southern Italy. Microorganisms 9, 1656. https://doi.org/10.3390/ microorganisms 9081656

77. Tito RY, Chaffron S, Caenepeel C, Lima-Mendez G, Wang J, Vieira-Silva S et al (2019) Population-level analysis of Blastocystis subtype prevalence and variation in the human gut microbiota. Gut 68(7): 1180-1189. https://doi.org/10.1136/ gutjnl-2018-316106

78. Yason JA, Liang YR, Png CW, Zhang Y, Tan KSW (2019) Interactions between a pathogenic Blastocystis subtype and gut microbiota: in vitro and in vivo studies. Microbiome 7(1): 1-13. https:// doi.org/10.1186/s40168-019-0644-3.

79. Poulsen CS, Efunshile AM, Nelson JA, Stensvold CR (2016) Epidemiological Aspects of Blastocystis Colonization in Children in Ilero Nigeria. Am J Trop Med Hyg 95:175-9. https://doi.org/10. 4269/ajtmh.16-0074

80. Sarinee J, Porntip P, Kookwan S, Somchai P, Arunnee S, Chotechana W, Wachanan W, Hisao Y (2013) Subtype identification of Blastocystis spp isolated from patients in a major hospital in northeastern Thailand. Parasitol Res 112:1781-6. https://doi.org/ 10.1007/s00436-012-3218-x

81. Deng L, Tay H, Peng G, Lee JWJ, Tan KSW (2021) Prevalence and molecular subtyping of Blastocystis in patients with Clostridium difficile infection. Singapore Parasites Vectors 14:277. https:// doi.org/10.1186/s13071-021-04749-8

Publisher's Note Springer Nature remains neutral with regard to jurisdictional claims in published maps and institutional affiliations. 